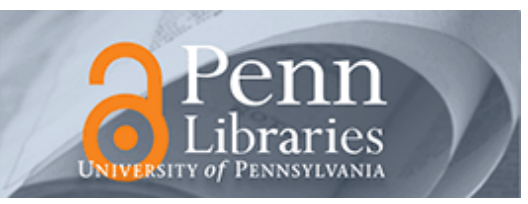

University of Pennsylvania

ScholarlyCommons

May 2006

\title{
Image Sensor with Focal Plane Extraction of Polarimetric Information
}

\author{
Viktor Gruev \\ University of Pennsylvania \\ Jan Van der Spiegel \\ University of Pennsylvania, jan@seas.upenn.edu \\ Nader Engheta \\ University of Pennsylvania, engheta@seas.upenn.edu
}

Follow this and additional works at: https://repository.upenn.edu/ese_papers

\section{Recommended Citation}

Viktor Gruev, Jan Van der Spiegel, and Nader Engheta, "Image Sensor with Focal Plane Extraction of Polarimetric Information", . May 2006.

Copyright 2007 IEEE. Reprinted from Proceedings of the IEEE International Symposium on Circuits and Systems (ISCAS), May 2006, pages 213-216.

Publisher URL: http://dx.doi.org/10.1109/ISCAS.2006.1692560

This material is posted here with permission of the IEEE. Such permission of the IEEE does not in any way imply IEEE endorsement of any of the University of Pennsylvania's products or services. Internal or personal use of this material is permitted. However, permission to reprint/republish this material for advertising or promotional purposes or for creating new collective works for resale or redistribution must be obtained from the IEEE by writing to pubs-permissions@ieee.org. By choosing to view this document, you agree to all provisions of the copyright laws protecting it.

This paper is posted at ScholarlyCommons. https://repository.upenn.edu/ese_papers/245

For more information, please contact repository@pobox.upenn.edu. 


\title{
Image Sensor with Focal Plane Extraction of Polarimetric Information
}

\author{
Abstract \\ A novel focal plane imaging sensor capable of real time extraction of polarization information is \\ presented. The imaging system consists of a photo array of 256 by 256 linear current mode active pixel \\ sensors (APS). Analog processing circuitry is included at the focal plane for noise suppression and \\ computation of the Stokes parameters. The imaging sensor was fabricated in $0.18 \mu \mathrm{m}$ process with $10 \mu \mathrm{m}$ \\ pixel pitch and $75 \%$ fill factor. An array of micro polarizer is designed and fabricated separately and will be \\ mounted on top of the imaging array. Simulation results of the imaging sensor are presented.

\section{Keywords} \\ CMOS imager, Image sensor, current-mode, polarization extraction, Stokes parameters

\section{Comments} \\ Copyright 2007 IEEE. Reprinted from Proceedings of the IEEE International Symposium on Circuits and \\ Systems (ISCAS), May 2006, pages 213-216. \\ Publisher URL: http://dx.doi.org/10.1109/ISCAS.2006.1692560 \\ This material is posted here with permission of the IEEE. Such permission of the IEEE does not in any way \\ imply IEEE endorsement of any of the University of Pennsylvania's products or services. Internal or \\ personal use of this material is permitted. However, permission to reprint/republish this material for \\ advertising or promotional purposes or for creating new collective works for resale or redistribution must \\ be obtained from the IEEE by writing to pubs-permissions@ieee.org. By choosing to view this document, \\ you agree to all provisions of the copyright laws protecting it.
}




\section{Image Sensor With Focal Plane Extraction of Polarimetric Information}

\author{
Viktor Gruev, Jan Van der Spiegel and Nader Engheta \\ Electrical and Systems Engineering Department \\ University of Pennsylvania \\ Philadelphia, Pennsylvania \\ \{vgruev,jan\}@seas.upenn.edu, engheta@ee.upenn.edu
}

\begin{abstract}
A novel focal plane imaging sensor capable of real time extraction of polarization information is presented. The imaging system consists of a photo array of 256 by 256 linear current mode active pixel sensors (APS). Analog processing circuitry is included at the focal plane for noise suppression and computation of the Stokes parameters. The imaging sensor was fabricated in $0.18 \mu \mathrm{m}$ process with $10 \mu \mathrm{m}$ pixel pitch and $75 \%$ fill factor. An array of micro polarizer is designed and fabricated separately and will be mounted on top of the imaging array. Simulation results of the imaging sensor are presented.
\end{abstract}

\section{INTRODUCTION}

Polarization vision contains important information about the imaged environment, such as surface shapes, curvature and material properties, which are ignored with traditional imaging systems [1]. Several species of invertebrate, such as cuttlefish, honeybees, desert ants, and others, rely on contrast enhancement using polarized vision, which is a vital survival mechanism in optically scattering media [2],[3]. The human eye perceives visual information in terms of color and intensity but it is blind to polarization. Hence, we are developing an imaging system capable of extracting polarization information from the imaged environment in real time and presenting the polarization information in parallel with the intensity information. This sensory system integrates imaging, micro-polarization array and polarization processing at the focal plane.

A survey of the literature reveals that most polarization sensitive imaging systems compute contrast enhancement information [4]-[7]. This information is extracted by either temporally sampling two images filtered with two orthogonal polarized filters [4][5][7] or integrating two orthogonal polarized filters over two neighboring photo elements [6]. The contrast extraction information is computed either on a DSP/CPU or at the focal plane with translinear circuits. Usual tradeoffs in these systems are reduction of frame rate vs. reduction of the spatial resolution in the latter systems. Incorporating pixel pitch matched polarization filters at the focal plane has been achieved using birefringence materials or thin film polarizers [6].

Although these sensory systems are directly inspired from biological systems (see, e.g. [2], [8]), they present limited polarization information in scattered media, such as fog, under water imaging and others [9]. In contrast, complete polarization information tends to be far more complex and its computational demands prevent real time extraction. These complex polarization properties are fully described by the fundamental equations known as the Stokes parameters [1]. In order to fully determine the Stokes parameters of natural (polychromatic) light, the scene must be sampled with three different polarization filters.

A micro-polarization array with three spatially distributed polarizers was fabricated and described by Guo et al. [10]. Manipulation of polymer polarization filters in order to create a micro-polarizer array was described in the patent by Faris [11]. One of the main challenges in manipulating a relatively thick polymer polarizing filter is the patterning and etching of the structures within $1 \mu \mathrm{m}$ accuracy. The thickness of commercially available polymer polarization thin films varies between $10 \mu \mathrm{m}$ to $20 \mu \mathrm{m}$, which creates problems when standard etching techniques employed in the semiconductor industry are used to create micro structures on the order of $10 \mu \mathrm{m}$ or less.

This paper describes a complete imaging system capable of extracting Stokes parameters of a linearly polarized light in real time. The paper is organized as follows. In Section 2, theoretical background of light polarization is presented and an alternative form for the Stokes equations suitable for focal plane implementation is discussed. Section 3 presents an overview of the micro polarizer array and the image sensor architecture. Section 4 briefly explains the focal plane computation of the Stokes parameters. Concluding remarks are summarized in Section 5.

\section{OVERVIEW OF POLARIZATION AND STOCKS PARAMETERS}

Polarization is a phenomenon observed in transverse waves. These are waves that vibrate in a direction perpendicular to their direction of propagation. Since light is a transverse wave, it can be represented as a sum of waves vibrating in (generally partially) random directions perpendicular to the line of propagation. If the vibration is consistently in a particular direction, the light is linearly polarized. Partial polarization of light usually occurs once 
the unpolarized light is reflected from a given surface or it has passed through a polarization filter.

The electric-field vector of a light wave traveling in the $z$ direction can be represented as a composition of the electric-field vectors oscillating in the $x-y$ plane.

$$
\begin{aligned}
& E=\mathbf{E}_{\mathbf{x}}+\mathbf{E}_{\mathbf{y}}= \\
& =\hat{\boldsymbol{x}} E_{x} \cos \left(\psi+\phi_{x}\right)+\hat{\boldsymbol{y}} E_{y} \cos \left(\psi+\phi_{y}\right)
\end{aligned}
$$

In equation (1), $\hat{\boldsymbol{x}}$ and $\hat{\boldsymbol{y}}$ are unit vectors along the $\mathrm{x}$ and y axes, $\psi=\omega t$ with $\omega$ being the radian frequency, $\phi_{x}$ and $\phi_{y}$ are the phases, while $E_{x}$ and $E_{y}$ are the magnitude of the electric-field in the $x$ and $y$ direction respectively. Depending on the relative difference between the phase $\phi_{x}$ and $\phi_{y}$, the transverse wave can be elliptically polarized if $\phi_{x}-\phi_{y}=$ constant, circularly polarized if $\phi_{x}-\phi_{y}=\pi / 2$ or linearly polarized if $\phi_{x}-\phi_{y}=0$.

The Stokes parameters presented by equations 2 through 5 describe fully the polarization state of the electric-field vector $E$.

$$
\begin{aligned}
& S_{0}=<E_{x}^{2}>+<E_{y}^{2}> \\
& S_{1}=<E_{x}^{2}>-<E_{y}^{2}> \\
& S_{3}=2<E_{x} E_{y} \cos \left(\phi_{x}-\phi_{y}\right)> \\
& S_{3}=2<E_{x} E_{y} \sin \left(\phi_{x}-\phi_{y}\right)>
\end{aligned}
$$

These equations are the traditional representation of the Stokes parameters. Following the Stokes equations, the polarization state can also be described if the following three quantities are known: $E_{x}, E_{y}$ and $\phi_{x}-\phi_{y}$. After some simple algebraic manipulations one can write:

$$
\begin{aligned}
& S_{0}=I_{t} \\
& S_{1}=2 I\left(0^{\circ}, 0\right)-I_{t} \\
& S_{2}=2 I\left(45^{\circ}, 0\right)-I_{t} \\
& S_{3}=I_{t}-2 I\left(45^{\circ}, \pi / 2\right)
\end{aligned}
$$

where $I_{t}$ is the total intensity; $\mathrm{I}\left(0^{0}, 0\right)$ is the intensity of the e-vector filtered with a 0 degree polarizer and no phase compensation between the $\mathrm{x}$ and $\mathrm{y}$ components; $\mathrm{I}\left(45^{\circ}, 0\right)$ is the intensity of the e-vector filtered with a 45 degree polarizer and no phase compensation as above; and $\mathrm{I}\left(45^{0}, \pi / 2\right)$ is the intensity of the e-vector filtered with a 45 degree polarizer and $\pi / 2$ phase compensator between the $\mathrm{x}$ and y components. The first three Stokes parameters fully describe the polarization of light with two linearly polarized intensities and the total intensity of the e-field vector. The fourth parameter describes the excess of right-circularly polarized component over the left-circularly polarized portion, which is less common in man-made and natural wave sources and it is not of interest in this application. It is important to point out that for a partially polarized polychromatic light in nature, the measurement of the fourth Stokes parameter is not essential, since in these scenarios the phase between the two orthogonal components of

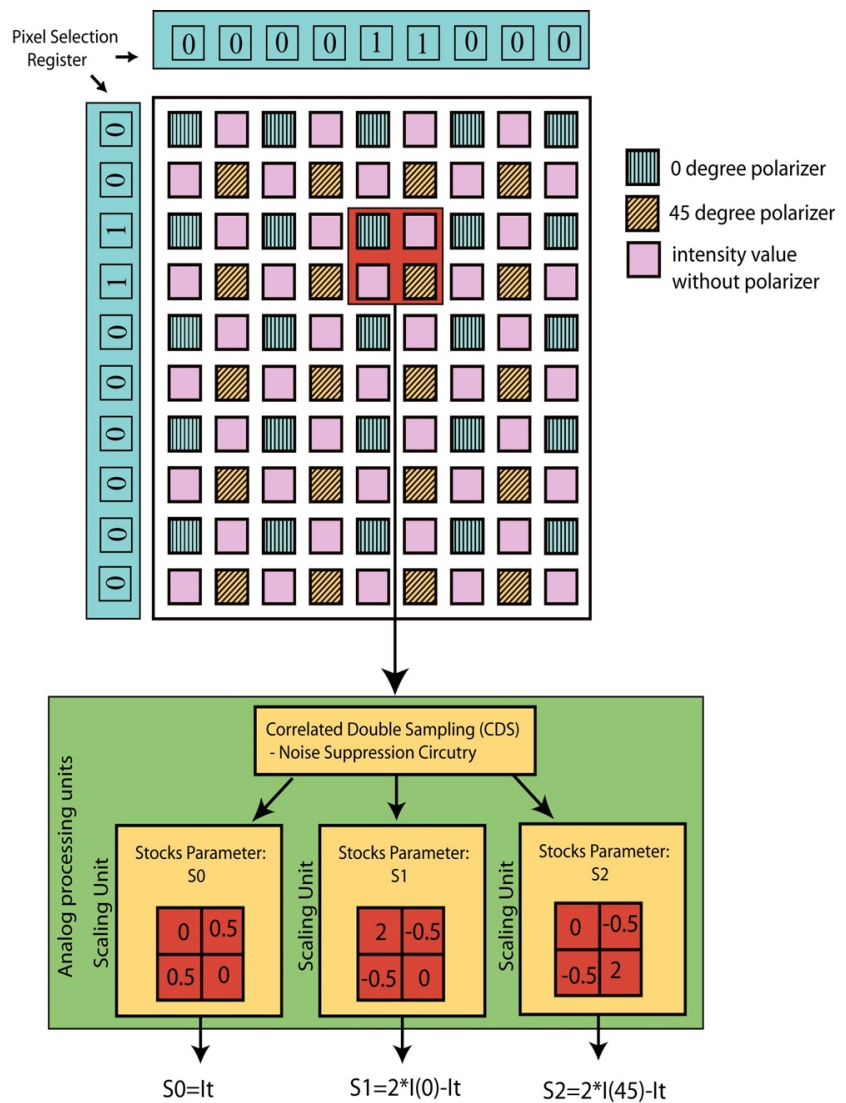

Figure 1: Block diagram of a complete focal plane polarization imaging system

polarization is usually statistically random. Therefore, for natural scenes, the first three Stokes parameters are usually of more interest, and thus 3 independent measurements are needed in order to find the first three Stokes parameters.

In order to fully describe the polarization state of light in nature, three linear polarized projections or two linear polarized projections in combination with the total intensity are needed. The latter method is preferred for focal plane implementation since it only requires two thin film polarizers offset by 45 degrees, patterned and placed on top of each other. The total thickness of the two layer micro polarizer array can be around $20 \mu \mathrm{m}$, if a commercially available thin film polarizer is used [12].

\section{IMAGING SENSOR AND MICRO-POLARIZER ARRAY ARCHITECTURAL OVERVIEW}

An overview of the complete polarimetric imaging system is presented in Figure 1. The imaging sensor consists of a 256 by 256 photo pixels array, reset and addressing registers in both horizontal and vertical directions, a correlated double sampling unit and a digitally controlled analog processing unit. Two thin film polarizers are individually patterned and deposited on top of the imaging array in order to form the pattern presented in Figure 1. The micro fabrication steps necessary for patterning the thin film polarizers are described in [12]. The thin film patterns and 


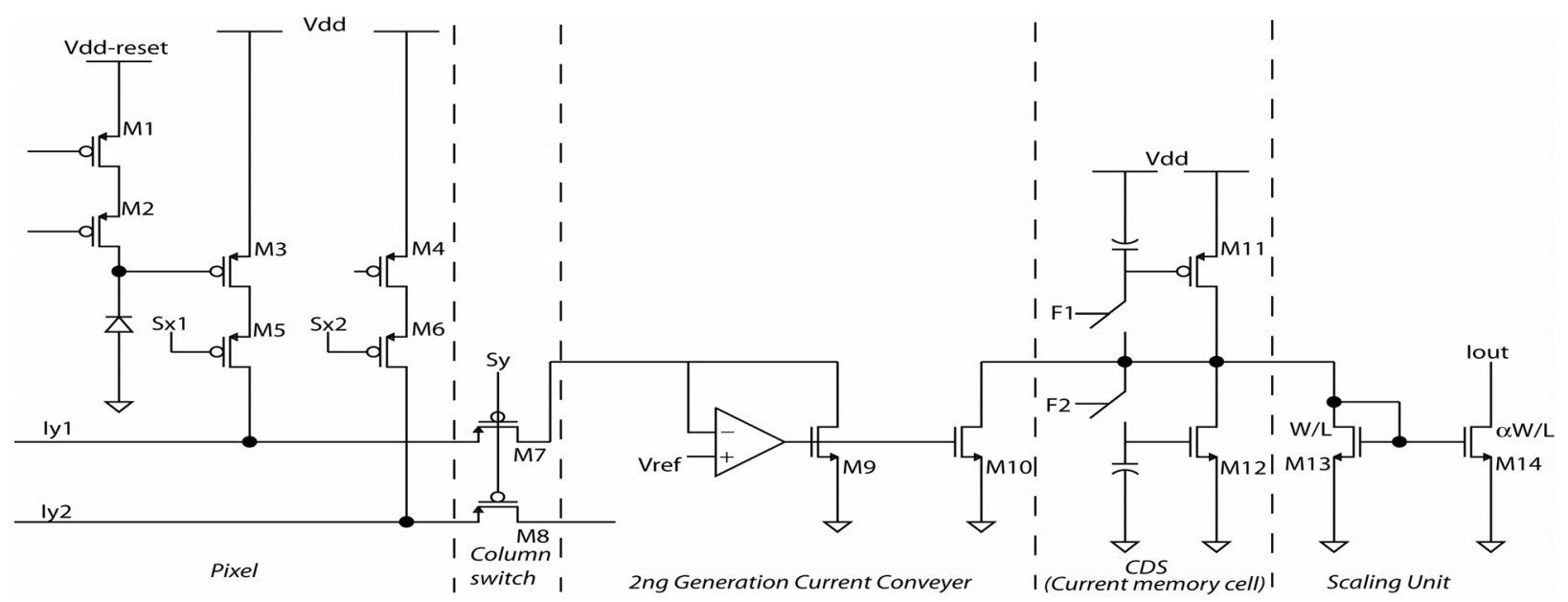

Figure 2: Complete circuitry of the pixel, current conveyer, CDS and scaling unit.

the photo pixel architecture allow for block parallel access of 2 by 2 pixels neighborhood. The polarization state of the pixel neighborhood is computed by scaling individual pixels according to the first three Stokes equation (6) though (8) (Figure 1).

The photo pixel is composed of transistors M1 through M6 (Figure 2). Transistors M1 and M2 control the operational mode of the photodiode. These two transistors allow individual pixels to be reset sequentially. Hence, a single noise correction circuitry is used for the entire array and it bypasses the column fixed pattern noise problems which are associated with column parallel read out. Transistors M3 and M4 operate as linear transconductance amplifiers and they linearly convert the integrated voltage on the photodiode into two output currents. The output photo currents are presented on two separate column busses, labeled $I_{\mathrm{y} 1}$ and $\mathrm{I}_{\mathrm{y} 2}$, which are individually addressed via transistors M5 and M6.

The linear photo current conversion is achieved by pinning the drain voltage of transistors M3 and M4 to $\mathrm{V}_{\mathrm{dd}}-\Delta$ voltage, while the reset voltage $V d d_{-}$reset is fixed to $\mathrm{V}_{\mathrm{dd}}-\Delta$ $\mathrm{V}_{\text {th. }}$. Therefore, during the reset period of the pixel, the gate voltage of transistor M3/M4 will be a threshold voltage bellow the drain voltage and transistor M3/M4 operates in the linear mode. During the integration period, the gate voltage of transistors M3 and M4 will further discharge and transistors M3 and M4 will remain in the linear mode. Switch transistors M5 through M8 are designed with high aspect ratios in order to decrease the voltage drop across these switches and therefore retain linear output current characteristics of the photo pixel.

The drain voltage of transistor M3/M4 is pinned to $\mathrm{V}_{\mathrm{dd}}-\Delta$ via the second generation current conveyer circuit shown in Figure 2 [13]. This circuit is composed of a two-stage operational amplifier with a Miller compensation capacitor operated in a negative feedback mode via transistor M9. The gain bandwidth product of the operational amplifier is $50 \mathrm{MHz}$ and $\mathrm{DC}$ gain $\mathrm{A}_{0}$ is $80 \mathrm{~dB}$. Due to its negative feedback configuration, the negative terminal of the op amp is pinned to $\mathrm{V}_{\text {ref }}=\mathrm{V}_{\mathrm{dd}}-\Delta$. The input resistance of the current conveyer is estimated by equation (10), in which $g_{m 9}$ is the transconductance of M9.

$$
r_{i n}=\frac{1}{q_{m 9} A_{0}}
$$

The minimum output current from the photo pixel is $3 \mu \mathrm{A}$ during the reset interval and the input resistance of the current conveyer is around $10 \Omega$. The input capacitance of the current conveyer consists of 256 gate-to-drain overlap capacitances plus the line capacitance of the metal bus, which is $2.5 \mathrm{~mm}$ long. With a total input capacitance of around $100 \mathrm{fF}$, the time constant of the current conveyer circuit is $1 \mathrm{~ns}$ or operational bandwidth of $1 \mathrm{Grad} / \mathrm{s}$ or 159Mhz. The operational amplifier further improves the tracking of the input voltage of the current conveyer as the input current is increased. The variations of the input terminal of the current conveyer over $10 \mu \mathrm{A}$ input range is less then $1 \mathrm{uV}$.

The noise suppression circuitry is based on a current memory cell described in [14]. The memory cell is composed of a coarse and a fine sub-memory cell. During the memorization stage of the coarse memory cell, charge injection error dependent on the input current level is introduced. These signal dependent charge injections are memorized in the fine memory cell and subtracted from the coarse memory cell. The final memorized current can replicate the original current with 12 bit accuracy.

The noise suppression of the photo pixel is performed in two steps. Initially the integrated photo current is memorized in the memory cell. Then the pixel is reset and the reset photo current is automatically subtracted from the integrated photo current. The final current output is independent of the voltage threshold variations of transistor M3 and M4 [15].

The last part of the processing unit is the digitally controlled analog processing unit. In this unit, the output current from the CDS unit is first replicated three times and then scaled accordingly to the Stokes equations. The scaling coefficient is represented as $\alpha \mathrm{W} / \mathrm{L}$ in Figure 2. Regulated cascaded mirrors are used throughout the processing unit allowing for better computational precision. 
Figure 3 presents a simulation of the circuit shown in Figure 2. The top graph present the current output from the pixel i.e. the drain current from transistor M3. The six doted lines present current output at different light levels. Hence, for stronger light intensities the discharge sloop is larger. At around $4 \mu \mathrm{sec}$, the pixel is reset and the reset current level is $3.5 \mu \mathrm{A}$. The bottom trace represents the final output of the circuit in Figure 2. At time F1, the coarse memory cell is sampled and at time F2 the fine memory cell is sampled in the CDS circuit. The final output is available after time F2. The different traces represent the final output at different light intensities. From this simulation, we observe that the CDS circuitry operates with 12 bit accuracy.

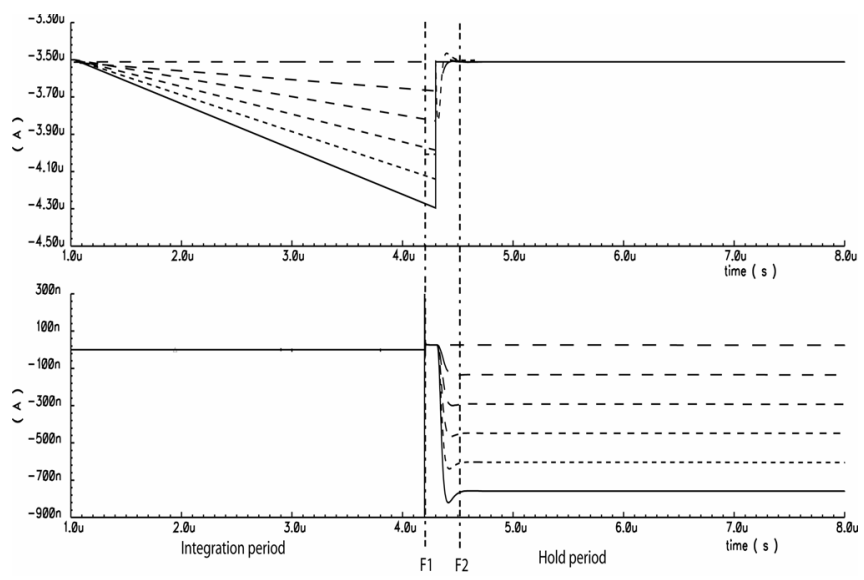

Figure 3: Simulation results of pixel linear output photocurrent and noise suppression circuitry for different light intensities.

\section{Algorithmic COMPUtation OF THE StOKes PARAMETERS}

The architectural design of the photo pixel and the addressing circuitry (Figure 1), allow for block-parallel access of 2 by 2 photo pixels. The circuitry shown in Figure 2 is applied to all four pixels within the window of polarimetry computation. The outputs from all four pixels are connected together in order to complete the computation of the Stokes parameters. The computation can be generalized by equation (11).

$$
S_{x}=\sum_{i=1}^{4} a_{i} I_{i} \text { for } \mathrm{x}=0 \text { to } 1
$$

where $S_{x}$ is the Stokes parameter, $I_{i}$ and $a_{i}$ are the photo current and scaling coefficient of the i-th pixel in the 2 by 2 neighborhood. Since there are three identical computational units, all three Stokes parameters are computed in parallel. A noise suppressed intensity image is presented outside the chip as well.

Table I shows the general characteristics of the chip. The simulated precision of the CDS unit is $12 \mathrm{bits}$ and processing bandwidth of $159 \mathrm{MHz}$. Another important aspect of the design is the high linearity of the photo pixel due to the high aspect ratios of the switch transistors and high accuracy of the second generation current conveyer.

\begin{tabular}{|c|c|}
\hline Technology & $0.18 \mu \mathrm{m} \mathrm{Nwell}$ \\
\hline No. Transistors & $800 \mathrm{~K}$ \\
\hline Array Size & $256 \times 256$ \\
\hline Pixel Size & $10 \mu \mathrm{m} \times 10 \mu \mathrm{m}$ \\
\hline Chip Size & $3.1 \mathrm{~mm} \times 3.3 \mathrm{~mm}$ \\
\hline CDS unit precision & $12 \mathrm{bits}$ \\
\hline Fill Factor & $75 \%$ \\
\hline Processing Circuitry & $159 \mathrm{MHz}$ \\
\hline
\end{tabular}

Table I: Chip summary

\section{ACKNOWLEDGMENT}

This work is support in part by U.S. Air Force Office of Scientific Research (AFOSR) grant number FA9550-05-10052 and a MOSIS research grant.

\section{REFERENCES}

[1] M. Born and E. Wolf, Principles in Optics, 3rd ed. New York: Pergamon, 1965.

[2] T. Labhart, "Polarization opponent interneurons in the insect visual system," Nature, 331, pp. 435-437, 1988.

[3] T. H. Waterman, "Polarization sensitivity" in The Handbook of Sensory Physiology vol. VII/6B Vision in Invertebrates, edited by $\mathrm{H}$. Autrum, Springer-Verlag, New York, 1981.

[4] L. B. Wolff and T. A. Mancini, "Liquid crystal polarization camera," in Proceedings of the IEEE Workshop on Applications of Computer Vision, pp. 120-127, 1992.

[5] Wolff, L.B.; Mancini, T.A.; Pouliquen, P.; Andreou,A.G. "Liquid crystal polarization camera," IEEE Transactions on Robotics and Automation, Vol. 13,No 2, pp.195-203, 1997.

[6] Andreou, A.G.; Kalayjian, Z.K "Polarization imaging: principles and integrated polarimeters," IEEE Sensors Journal, Vol. 2, No. 6, pp.:566-576, 2002.

[7] J. S. Tyo, E. N. Pugh, Jr., and N. Engheta, "Colorimetric Representations for Use with Polarization-Difference Imaging of Objects in Scattering Media," in Journal of Optical Society of America A, Vol. 15, No. 2, pp. 367-374, 1998.

[8] M. P. Rowe, N. Engheta, S. S. Easter, and E. N. Pugh, Jr., "Graded Index Model of a Fish Double Cone Exhibits Differential Polarization Sensitivity," in Journal of Optical Society of America A., Vol. 11, No. 1, pp. 55-70, January 1994.

[9] J. N. Lythgoe and C. C. Hemmings, "Polarized Light and Underwater Vision," Nature, 213, pp. 893-894, 1967.

[10] J. Guo and D. Brady, "Fabrication of high-resolution micropolarizer array," Optical. Engineering, Vol. 36, No. 8, pp. 2268-2271, 1997.

[11] S. M. Faris, "Methods for manufacturing micropolarizers," U.S. patent 5,327,285,July 1994.

[12] V. Gruev, K. Wu, J. Van der Spiegel and N. Engheta, "Micropolarization array fabrication", unpublished.

[13] A.M. Ismail and A.M. Soliman, "Wideband CMOD current conveyer," IEE Electronics Letters, Vol 34, No 25, pp.2368-2369, 1998.

[14] J. Hughes and K. Moulding, "S'I: the seamless S21 switched current cell", ISCAS'97, pp. 113-116, June 1997.

[15] V. Gruev, R. Etienne-Cumigns and T. Horiuchi, "Linear current mode imager with low fix pattern noise," ISCAS 2004. 